\title{
Scbillers Didbtungen
}

vor der chriftlichen Gemeinde.

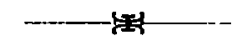

calo Deutlahe deutlch und gros empfinden, rüftet man tich bereits auf das Schillerfeft im Mai 1905. Hus der (alebmut der Erinnerung an Teinen allz̧uírühen Cod wird die Seele unieres Volkes Tich erbeben żu um to lebendigerer freude an all dem Shönen und Gerrlichen, was Tein rieblingsdichter vollbringen durfte.

Hber Sdiller ift viel mebr gewelen als ein Shöpfer ätthetilder שerte. Er hat uns bereibert und gefördert auch in dem Geiligiten unteres Cebens, in unterem driftliden Befitziftande. caie wenig zwar die Religion als lolde in teinem đaefen zu Cage tritt, to ift doch lein ganjes poetilahes Schaffen eine pergeiltigende und veredelnde caleiterbildung des in dem calerke der Reformation uns eritandenen deutthen Glaubens.

ruther und Sdiller, - zwei to veridiedene Raturen, find im Plane der Vorlehung ein einz̧iger Gedanke; und dieler Gottesgedanke drängt auf eine Kirche, die auch den Didter als eine leudtende Cebensoffenbarung ibres 5 errn erfaBt und lid zu einem Chriftentum bekennt, in dem der religiös tiefe und innige rutbergeift und der bumaniftilo geklärte und hochgeitimmte Sdillergeift fich in eins verweben tollen.

Von dieter abberzeugung getragen, werde it demnähit eine gröBere Anz̧ahl der Diobtungen Sdillers auf der Ranzel bebandeln. Es werden das alio nidht literaturgeidichtlide und ätthetifoe Darbietungen lein, londern im Didterworte die Verkündigung Chrifti und teines Evangeliums. Jo) verweife auf beifolgendes Verzeidnis der mir bis Dfingiten zufallenden Gottesdienite und der darauf lich verteilenden Didtungen.

(Drukldreiben an die Mitglieder der Gemeinde - Towle an die Geittichen und Cebrer Bremens und an jebn deutldhe Blätter - aus den caleihnahtstagen 1904.) 
$D_{\text {as }}$ in um[tehender ,'Mitteilung" feinerzeit angefündigte $\mathfrak{W}_{\text {ert }}$ ber zwanzig Sdillerpredigten übergebe if hiermit benen, bie lie gehört haben, wie allen, bie ibre Serausgabe wün|hten. J') übergebe fie ben Freunben, bie in vielen Gunderten von warmen 3uitimmungen in Briefen und Beitungsartifeln mein Borbaben begrübten, und biete lie meinen zablreiden Gegnern an, aud allen benen, bie nođ abwartend bis zur Deroffentlidung Des Ganzen ihr Urteil zurüdgebalten haber.

$\mathfrak{U l}_{\mathrm{s}}$ in ben eriten Monaten Des Jabres fajt bie ganze Deutide Prefie von ben Prebigten in ber Insgariitirde zu Bremen Renntnis nabm, und baraufbin, bejonders aud nad Dem (Ers ¡jeinen zweier Prebigten in ber „(Chriftllđen WeIt", von evange= Iijळ=orthodoxer wie von fatholifđer Seite ein Sturm ber Ents rüjtung und bes zornigen Wiberipruds gegen mid) entfelfelt wurbe, hat man wohl ber Meinung 2 usdrud gegeben, es wäre bejier gewejen, id hätte jenes weitere Sreije aufmerfjam madjende Druđfjureiben unterlajien und bie Sache ftill, wie jonjtige Prebig. ten, in bem engen Bezirfe meiner liberalen Bremer Gemeinde verlaufen Iaffen.

Dazu war lie aber viel zu neu und aukerorbentliđ. So etwas barf ein Pfarrer nidt in ber Berborgenbeit jeiner vier

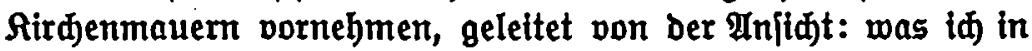
meiner (5emeinde tue, geht andere Gemeinden und bie Melt nidjts an. St. Insgaril ift bas Glied eines Rirdenganzen, ber

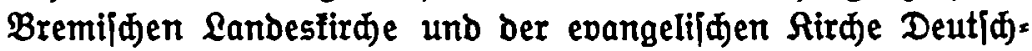
Iands. Jit erjere aud ein wenig realer, lebtere nod ein ganz ibeeller Begriff, fo fühlte id mid bod) in meinem ausge(prod)en 
firळliðen Bewußtjein verpflið)tet, über meine Tat in aller Dffenfidtlidfeit Der Gejamtheit Reden[daft zu geben.

3ubem lind bieje Sdillerpredigten nad inrem Charafter und ihrer 3wedanlage burdjaus nidst nur eine Gabe an meine

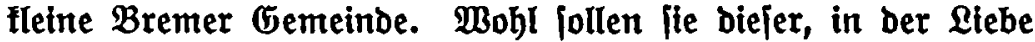

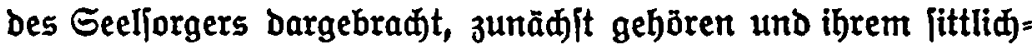
religiölen Leben bienen. Aber in ihrer inneriten Iendenz ent= itammen lie bem groben firdliđen Entwiălungsbrange ber Gegenwart. Dem wollen lie, mit einem flammenden $\mathfrak{B o r w a ̈ r t s}$ auf ihrer Stirn, 3iel und Mege weilend, einen Beitrag iteuern. Sie wollen mithelfen am $\mathfrak{A}$ ufbau einer neuen, berrlidjen Rirde Des beutiden (chriftentums. Bon $\mathfrak{A n j a n g}$ an lind dieje Predigten gedadt geweien als ein Mort an bie ganze beutiðe evangelijめe Chriltenheit, - jie in ihrem Sebnen und Derlangen, bie refor=

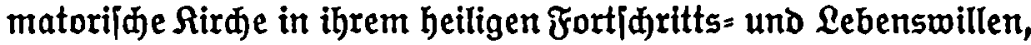
nid)t allein bie paar hunbert Meniđen vor ber Manzel, babe id) im Meden und Sd)affen vor $\mathfrak{A}$ ugen gehabt und ibrem Genius juðte iđ Worte 3u verieihen!

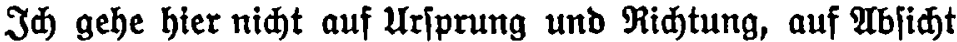
unb Beredtigung meiner Gdillerpredigten näber ein. Ja babe mid) Darüber in ben Reben jelbjt an mehreren Stellen in ver = (đ)ieben|ter Beziehung ausgejproden. (Fs find die Seiten: 9-20, $167-168,202-203,208-209,308,311,329-331,334,359$ bis $360,381-382,396$.

Da id meine Prebigten nid)t $3 \mathfrak{u}$ (đ)reiben pflege, fo find bie hier vorliegenden auf Grund von Stenogrammen - für bie iđ Dem Serm Stenographen ber Bürgeriđaft $\mathfrak{C}$. Riebau bier= mit verbindlidjt bante - entitanden. In ber Sadje, Dem (Geifte nad, ift nid)ts geändert. Nur hat bie rednerijđe frorm fiđ vielfad) in bie 'zum Leien geeignetere umgejest, und bas führt eine Berlängerung herbei. Dentr um bas, was Der Rebner in Ton und Saltung legt, oder was bie unmittelbare gottesoienit= liđe Stimmung im 3ubörer wirț, bei ber Druáfळrift wieber= 3ugeben, muß mand)es etwas weiter ausgeführt, reiđjer uno ergänzend geftaltet werben. Die 7., 18. und 19. Predigt haben einige burd) bie Widtigfeit ber Saゆe geforberte $3 u[a ̈ b e ~ e r=$ 
fabren; bas ganz Periōnlide in Der 1. Prebigt entitamimt ber Ausiprade in einem engeren Areije, Der biele Cinfügung in bas vorliegende Wert müniळte.

Charafter, Saltung und Ion der Predigten find jehr ver= iajieden, wohl teine ift barin ber anberen ähnlid). Einige ber

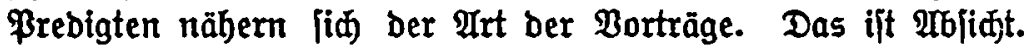
Es wäre mir leiđt gewejen, audi biejen eine mehr prebigtartige Geitalt zu geben. Erbaulid find fie, wie id Goffe, trob̧dem bod.

Ein Wort nod über die ben Prebigten vorausgebenden

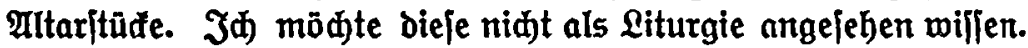
Eine jolde fennen wir in unjerer Sirde nidt. Der Gottesdienit belteht nur aus Gejang, Prebigt, Gebet und Segen; an Den altar geht ber (5eiftlide überbaupt nid)t. So habe id es vor= gefunber.

Seit längerer $3 e i t$ habe id barin eine \&nderung vorgenom= men. Das Bedürfnis, Der Gemeinde Den Reidtum, die Tiefe und Sđönbeit ber Geiligen Sđrift vorzufübren, veranlabte miđ, vor ber Predigt einiges am arltare vorzulejen. Daran fügte fid bann wohl ein (Gebet, und (đlieblid bildete (it) zwifden biejem am Mltar Dargebotenen und ber Prebigt ein febr enger 3ulammenhang. Wer darauf adten will, wird merten, daß Diejes Mitaritüa mit Ried und Prebigt ein organilaes Ganzes ausmađt, wo, wenn id jo [agen Darf, in funjtooller Stompojition eins bas anbere trägt und beutet. Das ber Predigt Boraus= gehende gehört aljo zu diejer und mub unbebingt mitgelejen werben.

So möge das Mert benn nun hinausgehen, geleitet von bem Sdillerworte aus einem leiner Briefe: „Ernjter, guter Wille ift eine grobe, bie iळönite (Eigeniळaft bes Geiltes. Der Exfolg liegt in einer böheren, unjidftbaren Sand. Nur die $\mathfrak{A b}=$ [iđt) gibt bem Mufwand von Sräften Mert. Und fo erbeben wit uns über Lob und Iabel ber Menjđjen!"

\&ugano, im Dftober 1905. 